\title{
REACTION PATH DESCRIPTION AND INTERNAL-MODE DYNAMICS OF MOLECULAR REARRANGEMENT IN $\mathrm{HO}_{2}$
}

\author{
Ch. ZUHRT, L. ZÜLICKE and F. SCHNEIDER \\ Zentralinstitute für physikalische Chemie, Rudower Chaussee 5, D-0-1199 \\ Berlin, Germany \\ X. CHAPUISAT \\ Laboratoire de Chimie Théorique (CNRS US 506), Bâtiment 490, Faculté \\ des Sciences, Université de Paris-Sud, 91405-Orsay, Cêdex, France
}

\begin{abstract}
After brief general remarks and definitions for the reaction path concept in view of formulating physically transparent and computationally feasible theoretical models, the $\mathrm{HO}_{2}$ system is studied for both the bimolecular exchange reaction and the intramolecular $\mathrm{H}$ transfer (isomerization). The analysis includes potential energy surface topography, reaction profiles and internal-mode dynamics.
\end{abstract}

KEY WORDS: Reaction, dynamics, rearrangment, $\mathrm{HO}_{2}$.

The theoretical treatment of molecular rearrangement processes, according to the standard methodology, is still a formidable task, in particular for polyatomic systems. In the last decade, following the pioneering work of Miller, Handy and Adams, ${ }^{1}$ much effort has been made to formulate the theory in a compact way retaining only those features of the problem which are dynamically essential. The basic idea is that some kind of reaction path (RP) must exist, since the molecular rearrangements are frequently local phenomena, whence the expectation that a reduced treatment should be possible by means of (i) a static (i.e. range) reduction to that part of the configuration space which is energetically relevant (the RP and its near environment); (ii) a dynamic (i.e. dimensional) reduction to that subspace of the configuration space which is dynamically relevant. ${ }^{2}$ The aim of the present paper is to illustrate such ideas for the simple system $\mathrm{HO}_{2}$.

\section{THE REACTION PATH CONCEPT: DEFINITIONS}

The RP for a molecular rearrangement is defined as the curve of least energy connecting, on the potential energy surface, two local minima via a first-order saddle point. ${ }^{3}$ The arc length of the RP, $s$, is properly identified with the reaction coordinate (RC), and the potential energy function along the RP, $U_{0}(s)$, is frequently called the reaction profile. 
If, along the RP, a local normal mode analysis is performed pointwisely, the non-zero vibrational frequencies, obtained by diagonalizing a properly projected Hessian matrix ${ }^{1}$ belong to the so-called transverse modes orthogonal to the RP. From the corresponding eigenvectors, the couplings of these modes to one another, to the RP and to the external modes (overall translation and rotation of the whole system) can be calculated. ${ }^{1}$

\section{$\mathrm{HO}_{2}$ : REACTION PATHS, REACTION PROFILES AND INTERNAL-MODE} DYNAMICS

In $\mathrm{HO}_{2}$, various dynamical regimes are possible: molecular vibrations, isomerization $\mathrm{HO}_{2} \rightleftarrows \mathrm{O}_{2} \mathrm{H}$, fragmentation into $\mathrm{H}+\mathrm{O}_{2}$ or $\mathrm{HO}+\mathrm{O}$, and bimolecular exchange, $\mathrm{H}+\mathrm{O}_{2} \rightleftarrows \mathrm{HO}+\mathrm{O}$. Our study uses the analytical potential energy function by Varandas (version II). ${ }^{4}$ In Figure 1, the reaction profile for the exchange process is presented showing, at $s=0$, the deep minimum for stable $\mathrm{HO}_{2}(-2.305 \mathrm{eV}$ with respect to $\mathrm{H}+\mathrm{O}_{2}$, respectively); the arrow indicates the top of the isomerization barrier. The transverse mode frequencies along the RP are plotted in Figure 2. Around the entrance channel saddle point, the upper curve, connecting the $\mathrm{O}_{2}$ vibrational frequency at low $s$ with the $\mathrm{OH}$ frequency at large $\mathrm{s}$, and the lower curve representing the bending frequency which goes to zero on both ends (free rotation of diatomic fragments), come close together. In this region, the two transverse modes interact strongly with one another and with the RP (see Figures 3 and 4). Three other peaks of the coupling of the bending transverse mode with the RP are clearly observed in Figure 4; they likewise indicate regions of strong curvature of the RP. These findings are in rough accordance with those previously obtained ${ }^{5}$ in using the potential of Melius and Blint. ${ }^{6}$

The same analysis for the isomerization RP gives the potential profile and the transverse mode frequencies presented in Figures 5 and 6, respectively. The frequencies in Figure 6 have been identified as bending (lower) and $\mathrm{OH}$ stretching (upper), respectively. They evolve smoothly along the isomerization RP. Strong intermode coupling and mode-RP coupling only occur in the vicinity of the $\mathrm{HO}_{2}$ absolute minimum.

After the above (static) analysis of the interaction potential and of the behaviour of the different modes in the RP representation, we have investigated the dynamics of $\mathrm{HO}_{2}$ by running classical trajectories for energies ranging from the vibrational ground state up to above the isomerization barrier but below the fragmentation energies: $-1.953 \mathrm{eV}<E_{\text {total }}<-0.68 \mathrm{eV}$. The initial conditions were chosen so as to fit normal vibrational states of $\mathrm{HO}_{2}$. The results have been Fourier analyzed by calculating the quantity.

$$
I(\omega)=(1 / 2 \pi) \lim _{T \rightarrow \infty}(1 / 2 T)\left|\int_{-T}^{+T} X(t) \exp (-\mathrm{i} \omega t) \mathrm{d} t\right|^{2}
$$

for the dynamical variable

$$
X(t)=r_{1}(t)+r_{2}(t)+r_{3}(t)
$$




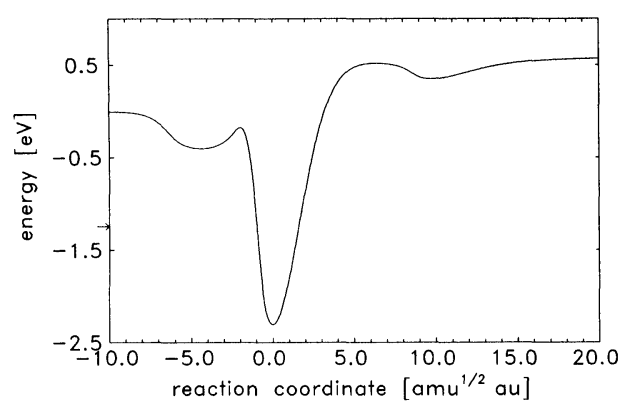

Figure 1 Potential energy profile along the RP for the $\mathrm{H}+\mathrm{O}_{2}$ exchange process.

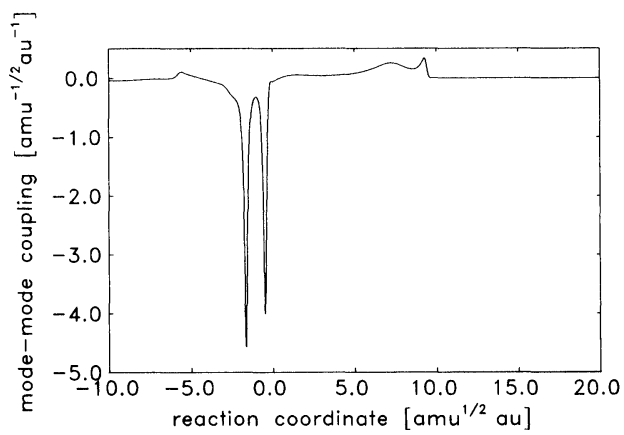

Figure 3 Coupling coefficient for the interaction between the two transverse modes for the $\mathrm{H}+\mathrm{O}_{2}$ exchange process.

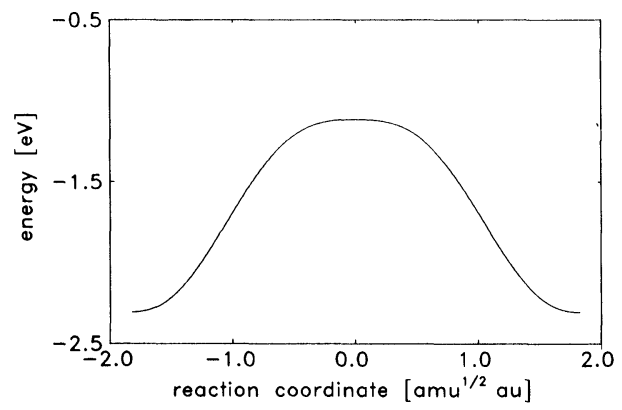

Figure 5 Potential energy profile along the RP for the $\mathrm{HO}_{2}$ isomerization process.

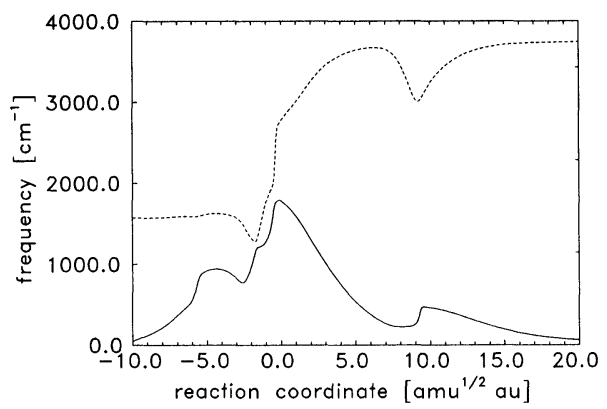

Figure 2 Transverse mode frequencies along the $\mathrm{RP}$ for the $\mathrm{H}+\mathrm{O}_{2}$ exchange process (dashedstretching mode, full-bending mode).

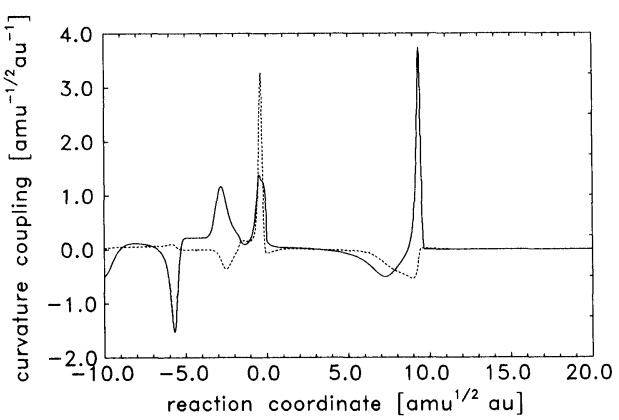

Figure 4 Coupling coefficients for the interaction of transverse modes with the $\mathrm{RC}$ for the $\mathrm{H}+\mathrm{O}_{2}$ exchange process (dashed-stretching mode, full-bending mode).

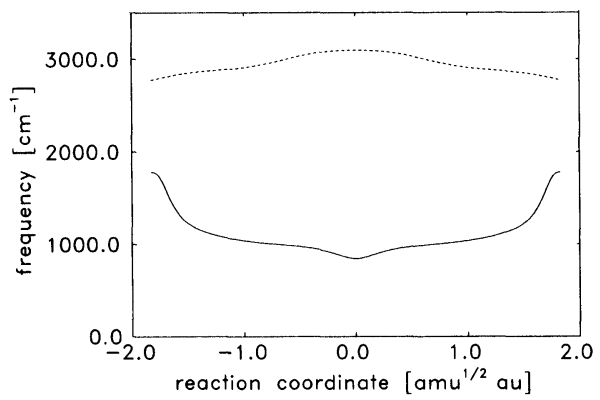

Figure 6 Transverse mode frequencies along the $\mathrm{RP}$ for the $\mathrm{HO}_{2}$ isomerization process (dashedstretching mode, full-bending mode). 
i.e. the sum of the three internuclear distances, so as to detect all internal motion frequencies. A few selected results are presented in Figure 7; the ordinate is for the quantity $\left\langle I^{1 / 2}(\omega)\right\rangle$, where the brackets denote the ensemble average over the initial vibrational phases.

For vibrational energies below the isomerization barrier $(1.191 \mathrm{eV})$ we have definitely a line spectrum corresponding to the three normal vibrations with quanta $1124 \mathrm{~cm}^{-1}, 1782 \mathrm{~cm}^{-1}$ and $2775 \mathrm{~cm}^{-1}$, respectively (see Figure $7 \mathrm{a}$ and b). Further excitation of the first vibrational mode only (which correlates with the isomerization $\mathrm{RP}$ ) leads to the onset of chaotic motion (Figure 7c). Nevertheless, some mode specificity is still observable in Figure 7c, whereas, in Figure 7d which is for about the same total energy but for a different distribution over the modes, the frequency bands are much broader, whence mode specificity is lost and strongly chaotic vibrational motion is established.
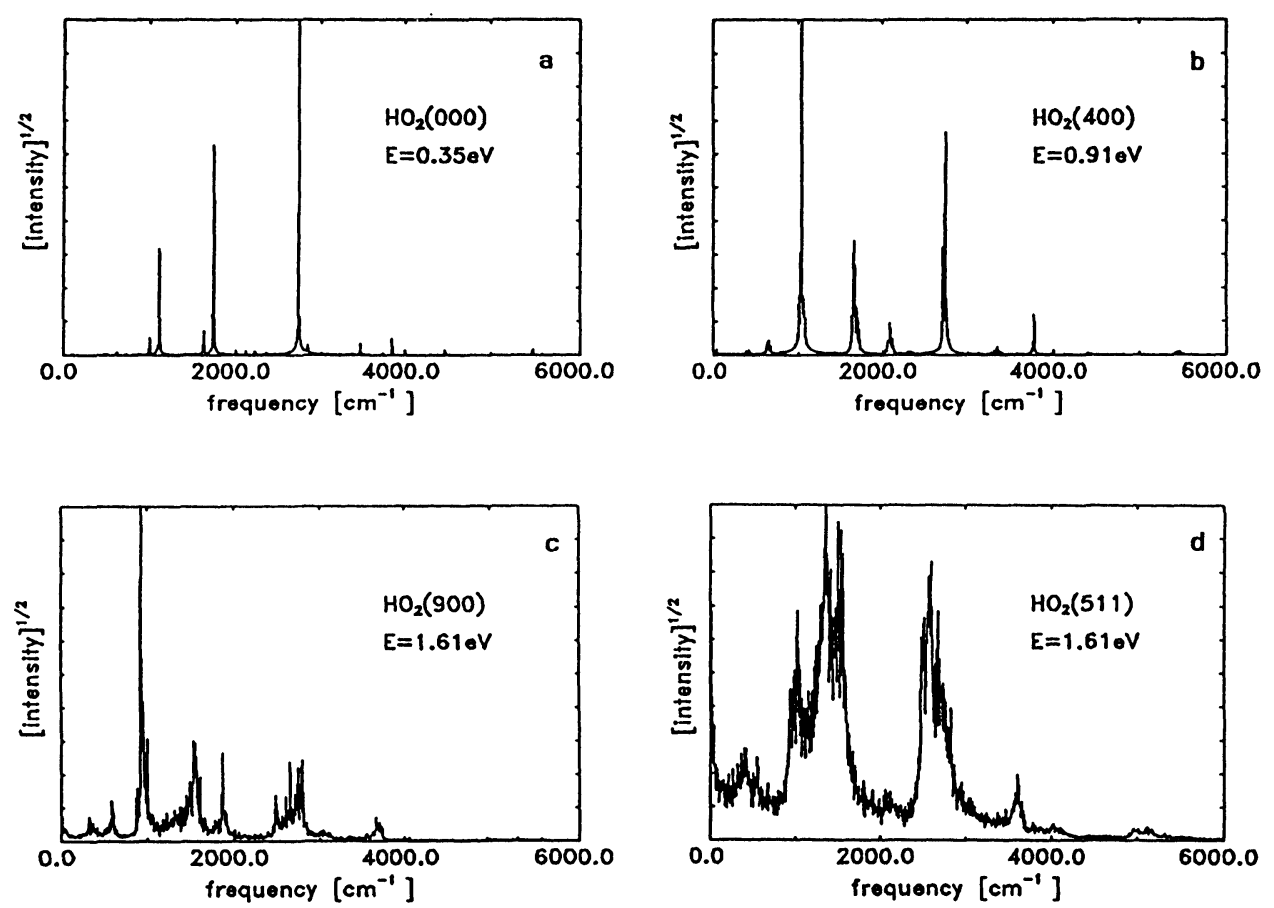

Figure 7 Spectral analysis of classical trajectories with initial conditions corresponding to normal vibrational states $\left(v_{1} v_{2} v_{3}\right)$ of $\mathrm{HO}_{2}$.

\section{CONCLUSIONS}

Studies of the potential energy surface topography and of the dynamical behaviour are important for analyzing the reactive properties of a molecular system in several respects: (1) The results provide the input data for the formulation of Reaction Path 
Hamiltonians. ${ }^{1,2,7,8}$ (2) The dynamical findings concerning mode coupling, energy exchange and onset of stochastic dynamics indicate whether it is possible to introduce dynamical simplifications like system-bath decomposition of the system, statistical approximations etc. ${ }^{9,2}$ Case studies as presented here can help to elucidate the main features of dynamical phenomena.

\section{References}

1. W. H. Miller, N. C. Handy and J. E. Adams, J. Chem. Phys. 72, 99 (1980).

2. L. Zülicke and A. Merkel, Int. J. Quantum Chem. 38, 191 (1990).

3. P. Pechukas, J. Chem. Phys. 64, 1516 (1976).

4. A. J. C. Varandas, J. Brandao and L. M. Quintales, J. Phys. Chem. 92, 3732 (1988).

5. F. Schneider, L. Zülicke and X. Chapuisat, Mol. Phys. 71, 17 (1990).

6. C. F. Melius and R. J. Blint, Chem. Phys. Lett. 64, 183 (1979).

7. A. Nauts and X. Chapuisat, Chem. Phys. 76, 349 (1983).

8. T. Carrington and W. H. Miller, J. Chem. Phys. 81, 3942 (1984).

9. W. H. Miller, J. Phys. Chem. 87, 3811 (1983). 